\title{
ON THE UNILATERAL EQUATION IN MATRICES*
}

\author{
BY \\ WILLIAM E. ROTH
}

The equation

(1)

$$
\sum_{i=0}^{p} A_{i} X^{p-i}=0,
$$

in which the coefficients $A_{i}, i=0,1,2, \cdots, p$, are $m \times n$ matrices having $m$ rows and $n$ columns of scalar elements, and where $X$ is an $n \times n$ matrix, is said to be unilateral $\dagger$ in that the known matrices $A_{i}, i=0,1,2, \cdots, p$, occur as factors on the left (or on the right) of each term of its left member. In case $m=n$ the equation was studied by Sylvester in several papers of a series that appeared largely in the Johns Hopkins University Circulars of 1883 and 1884, the Philosophical Magazine, and the Comptes Rendus de l'Académie de Sciences. $\ddagger$ The solution of particular types of equations in matrices that may be regarded as special cases under the above was further studied by Buchheim, Frobenius, Taber, Kreis, Cecioni, Wedderburn and others. $\$$ The restrictions adopted heretofore were such that the given

* Presented to the Society, August 29, 1929; received by the editors in August, 1929.

† Sylvester, Equations in matrices, Johns Hopkins University Circulars, vol. 3 (1884), p. 122, or Mathematical Papers, vol. IV, pp. 152-153.

$\ddagger$ See particularly On the three laws of motion in the world of universal algebra, Johns Hopkins University Circulars, vol. 3 (1884), pp. 33, 34, 57, or Mathematical Papers, vol. IV, pp. 146-151; Sur l'equation quadratique de Hamilton en quaternions ou en matrices du second ordre, Comptes Rendus, vol. 99 (1884), pp. 555-558, pp. 621-631, or Mathematical Papers, vol. IV, pp. 190-198. This paper will be referred to as E.Q.

$\S$ Buchheim, An extension of a theorem of Professor Sylvester's relating to matrices, Philosophical Magazine, vol. 22 (1886), p. 173.

Frobenius, Über lineare Substitutionen und bilineare Formen, Crelle's Journal, vol. 84 (1878), pp. 1-63; we shall refer to this paper as L.S. Also, Über die cogredienten Transformation der bilinearen Formen, Sitzungsbericht der königlichen Preussischen Akademie der Wissenschaften, 1896. This paper gives solutions of $X^{2}=A$ expressible as polynomials in $A$.

Taber, On the automorphic linear transformation of an alternate bilinear form, Mathematische Annalen, vol. 46 (1895), pp. 561-583.

Kreis, Contribution à la Theorie des Systèmes Linéaires, Zurich Thesis, 1906, and Auflösung der Gleichung $X^{m}=A$, Vierteljahrschrift der Naturforschenden Gesellschaft in Zurich, vol. 53 (1908), pp. 366-376.

Cecioni, Sopra alcune operazioni algebriche sulle matrici, Annali della Reale Scuola Normalè Superiore di Pisa, vol. 11 (1909), pp. 1-140.

Wedderburn, The automorphic transformation of a bilinear form, Annals of Mathematics, (2), vol. 23 (1923), pp. 122-134.

Roth, $A$ solution of the matric equation $P(X)=A$, these Transactions, vol. 30 (1928), pp. 579-596.

Franklin, Algebraic matric equations. This paper has not appeared in print. An abstract appears in the Bulletin of the American Mathematical Society, vol. 35 (1929), p. 7. 
equation might have at best matric coefficients every two of which are commutative. The only cases in which equation (1) has received attention as far as the writer is aware is in the algebra of quaternions and that given it by Sylvester.*

In the following pages we shall seek necessary conditions for the existence of a solution of (1) and at the same time we shall indicate how a solution, when it exists, may be computed. The literature gives very essential aids in the attack of this problem when solutions whose elements belong to a restricted field of rationality are sought, and we have the work of Cecioni $\dagger$ as an example of such an attack upon a related problem; we shall nevertheless consider only solutions, $X$, of the given equation whose elements belong to the complex number field of rationality. The results we derive will come from equation (1) which has the known matrices on the left of its terms, but with an entirely parallel procedure the proofs and theorems can be carried out for an $m \times m$ matrix as a solution of the equation where all $A$ 's occur on the right of the unknown.

The notation that will be adopted is essentially that employed in the literature. $\ddagger$ We shall use large italic letters to designate matrices having several columns, but when the matrices have a single column of elements we shall indicate the fact by using script capitals. The elements of the $m \times n$ matrix $A$ are the scalars $a_{i j}, i=1,2, \cdots, m, j=1,2, \cdots, n$, and with the convention just stated we may write

$$
A=\left(\mathcal{A}_{1}, \mathcal{A}_{2}, \cdots, \mathcal{A}_{n}\right),
$$

where $\mathcal{A}_{j}, j=1,2, \cdots, n$, is the matrix formed by the $m$ elements in the $j$ th column of $A$. The scalar elements of a matrix will always be indicated by lower case letters.

\section{Section I}

Suppose that the equation (1) has a solution $X$, which of necessity is an $n \times n$ matrix since we are interested particularly in the case where the degree, $p$, of (1) exceeds unity. Let the elementary divisors of $|X-\lambda I|$ be

$$
\left(x_{i}-\lambda\right)^{n_{i}} \quad(i=1,2, \cdots, s),
$$

where $\sum_{i=1}^{s} n_{i}=n$ and where $x_{i}, i=1,2, \cdots, s$, are not necessarily distinct. Then let

\footnotetext{
* E.Q., pp. 621-631.

† Cecioni, loc. cit.

$\ddagger$ Bocher, Introduction to Higher Algebra, 1907. Muth, Theorie und Anwendung der Elementartheiler.
} 
and

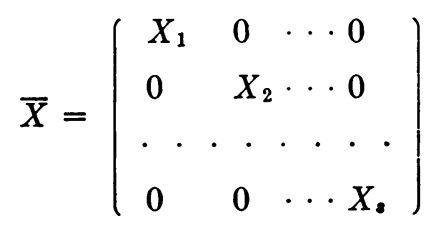

$$
X_{i}=\left(\begin{array}{cccccc}
x_{i} & 1 & 0 & \cdots & 0 & 0 \\
0 & x_{i} & 1 & \cdots & 0 & 0 \\
. & . & . & . & . & . \\
0 & 0 & 0 & \cdots & x_{i} & 1 \\
0 & 0 & 0 & \cdots & 0 & x_{i}
\end{array}\right) \quad(i=1,2, \cdots, s)
$$

where $X_{i}$ is an $n_{i} \times n_{i}$ matrix. ${ }^{*}$ The determinant $|\bar{X}-\lambda I|$ will then have the same elementary divisors that $|X-\lambda I|$ has and there consequently exists a non-singular $n \times n$ matrix, $Q$, such that

$$
X=Q \bar{X} Q^{-1} \cdot \dagger
$$

Hence equation (1) becomes

$$
\sum_{j=0}^{p} A_{j} Q \bar{X}^{p-i} Q^{-1}=0
$$

or, multiplying through by $Q$ on the right, we have

$$
\sum_{j=0}^{p} A_{i} Q \bar{X}^{p-j}=0 .
$$

Let

$$
\nu_{i}=n_{1}+n_{2}+\cdots+n_{i-1} \quad(i=1,2, \cdots, s),
$$

where $\nu_{1}=0$, and let $Q_{j}$ be the $m \times 1$ matrix formed by the $j$ th column of $Q$. With this we find that the matric equation giving the $n_{i}$ columns following the first $\nu_{i}$ of the members of (3) is

$$
\sum_{j=0}^{p} A_{j}\left(\mathcal{Q}_{\nu_{i+1}}, \mathscr{Q}_{\nu_{i}+2}, \cdots, \mathcal{Q}_{\nu_{i}+n_{2}}\right) X_{i}^{p-j}=0 .
$$

Now let

$$
A(\lambda)=\sum_{j=0}^{p} A_{j} \lambda^{p-\jmath}=\left(a_{h k}(\lambda)\right),
$$

where $\lambda$ is a scalar variable; the element $a_{h k}(\lambda), h=1,2, \cdots, m, k=1,2$, $\cdots, n$, is a polynomial in $\lambda$ whose degree does not exceed $p$. Also let

* Zero matrices of whatever dimensions will be given by 0 ; their horizontal and vertical orders will be clear from the expressions in which they occur.

† Bठcher, op. cit., p. 283. 


$$
A^{(r)}(\lambda)=\left(a_{h k}^{(r)}(\lambda)\right)
$$

where $a_{k k}^{(r)}(\lambda)$ is the $r$ th derivative of $a_{h k}(\lambda)$ with respect to $\lambda$. Since

$$
\begin{array}{r}
a_{h k}(\lambda) \equiv a_{h k}\left(x_{i}\right)+a_{h k}^{\prime}\left(x_{i}\right)\left(\lambda-x_{i}\right)+\frac{a_{h k}^{(2)}\left(x_{i}\right)}{2 !}\left(\lambda-x_{i}\right)^{2} \\
+\cdots+\frac{a_{h k}^{(p)}\left(x_{i}\right)}{p !}\left(\lambda-x_{i}\right)^{p}
\end{array}
$$

we are then permitted to write

$$
\begin{aligned}
A(\lambda) \equiv A\left(x_{i}\right)+A^{\prime}\left(x_{i}\right)(\lambda & \left.-x_{i}\right)+\frac{A^{(2)}\left(x_{i}\right)}{2 !}\left(\lambda-x_{i}\right)^{2} \\
& +\cdots+\frac{A^{(p)}\left(x_{i}\right)}{p !}\left(\lambda-x_{i}\right)^{p}
\end{aligned}
$$

Now

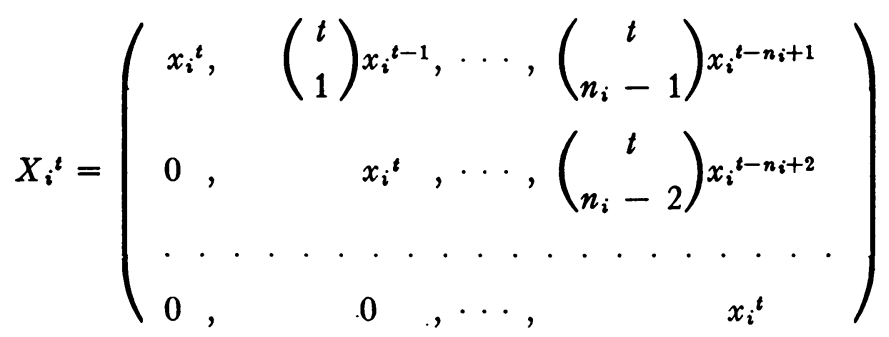

where $t$ is a positive integer and $\left(\begin{array}{l}t \\ j\end{array}\right)$ is the $(j+1)$ st binomial coefficient. For $X_{i}{ }^{0}$ we may write the unit $n_{i} \times n_{i}$ matrix. Because the elements $x_{i}$ are scalars they are commutative with $\mathscr{Q}_{j}, j=1,2, \cdots, n$, and we can then readily verify the fact that the first, second, $\cdots, n_{i}$ th columns of the members of (4) satisfy the equations

$$
\begin{aligned}
& A\left(x_{i}\right) \mathcal{Q}_{v_{i+1}}=0, \\
& A^{\prime}\left(x_{i}\right) \mathcal{Q}_{\nu_{i+1}}+A\left(x_{i}\right) \mathcal{Q}_{\nu_{i+2}}=0,
\end{aligned}
$$

$$
\frac{A^{\left(n_{i}-1\right)}\left(x_{i}\right)}{\left(n_{i}-1\right) !} 2_{v_{i}+1}+\frac{A^{\left(n_{i}-2\right)}\left(x_{i}\right)}{\left(n_{i}-2\right) !} 2_{\nu_{i}+2}+\cdots+A\left(x_{i}\right) 2_{\nu_{i}+n_{i}}=0
$$

Now by hypothesis $Q$ is a non-singular matrix, hence none of its columns can be a zero matrix. The first equation above is a system of $m$ linear and 
homogeneous equations* in the $n$ elements of $2_{v_{i}+1} \neq 0$; we can therefore conclude that the rank, $r_{i 1}$, of $A\left(x_{i}\right)$ is less than $n$. $\dagger$ When $m<n$ this condition is always satisfied by an arbitrary $x_{i}$. We shall henceforth speak of $x_{i}$, $i=1,2, \cdots, s$, as the characteristic values of $X$. We therefore have the following theorem. $\ddagger$

THEOREM I. The characteristic values, $x_{i}, i=1,2, \cdots, s$, of $X$, a solution of (1), must be such that the rank of $A\left(x_{i}\right)$ is less than $n$.

When $m<n$ this theorem gives us no information regarding the characteristic values of $X$.

Corollary. The number of distinct characteristic values of $X$ cannot exceed the number of distinct values of $\lambda$ for which the rank of $A(\lambda)$ is less than $n$.

The rank of $A\left(x_{i}\right)$ being $r_{i 1}$, then the number of linearly independent matrices, $2_{p_{i+1}}$, that satisfy the first equation of (6), is $n-r_{i 1}$.

THEOREM II. If $X$ is a solution of (1) whose characteristic determinant $|X-\lambda I|$ has the elementary divisors

$$
\left(x_{i}-\lambda\right)^{n_{i}} \quad(i=1,2, \cdots, s),
$$

the number of elementary divisors that are powers of the same linear factor $x_{i}-\lambda$ cannot exceed $n-r_{i 1}$, where $r_{i 1}$ is the rank of $A\left(x_{i}\right)$.

We shall now establish two theorems basing our reasoning on all $n_{i}$ equations of (6).

\section{SeCtion II}

$A(\lambda)$ is an $m \times n \lambda$-matrix for which we shall form an equivalent $\lambda$-matrix, $B(\lambda)$, by means of the following transformations:

1. The interchange of any two columns.

2. The multiplication of each element of a column of $A(\lambda)$ by the same constant not zero.

3 . The addition to the elements of one column of the products of corre-

* The rows of the matrix $\sum_{j=0}^{p} \lambda_{j} A_{j}$ may be regarded as linearly independent for all values of $\lambda_{j}, j=0,1,2, \cdots, p$, for if this matrix has less than $m$ linearly independent rows then the same linearly dependent rows may be dropped from all the matrices $A_{j}, j=0,1, \cdots, p$, without affecting the solution of (1) on the right in any way.

$\dagger$ Bốcher, op. cit., p. 47.

$\ddagger$ This theorem for the case $m=n$ was first announced by Sylvester, E.Q., p. 621 . He gives an interesting account of the growth of the ideas that led him to its discovery in Nature (Nov. 13, 1884, pp. 35-36). His proof is an intuitive one. A rigorous proof of a theorem slightly more general than Sylvester's was given by H. B. Phillips, Functions of matrices, Theorem I, American Journal of Mathematics, vol. 41 (1919), p. 287. 
sponding elements of another column by one and the same polynomial in $\lambda$.* The matrix $A(\lambda) Q$ is equivalent to $A(\lambda)$, since $Q$ is a non-singular matrix and the multiplication of $A(\lambda)$ by it on the right employs only the three transformations above. Let

where

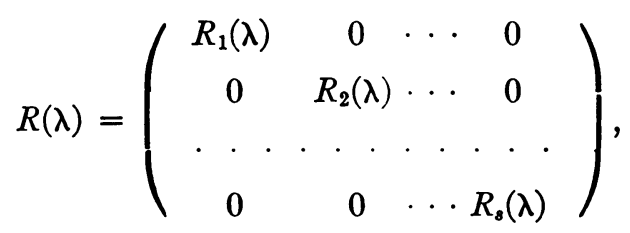

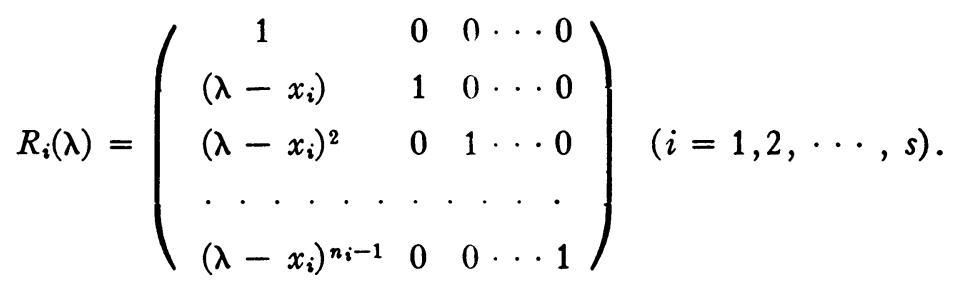

Then $|R(\lambda)|=1$ and $A(\lambda) Q R(\lambda)$ is again equivalent to $A(\lambda)$. If $A(\lambda)$ be replaced by its identically equal given by (5) and if the operations indicated by $A(\lambda) Q R(\lambda)$ be performed, we see by (6) that

$$
A(\lambda) Q R(\lambda) \equiv\left(B_{1}(\lambda), B_{2}(\lambda)_{o}, \cdots, B_{s}(\lambda)\right)
$$

where

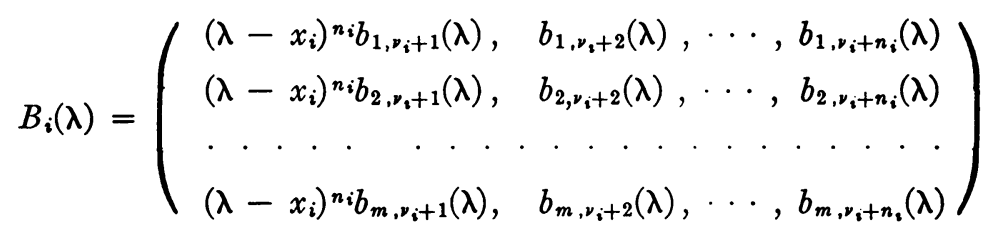

and the elements $b_{k_{i}, v_{i}+h}(\lambda), k=1,2, \cdots, m, h=1,2, \cdots, n_{i}$, are polynomials whose degree in $\lambda$ does not exceed $p$.

THEOREM III. If $X$ is a solution of (1) and if $|X-\lambda I|$ has the elementary divisors $\left(x_{i}-\lambda\right)^{n_{i}}, i=1,2, \cdots, s$, then the $\lambda$-matrix, $A(\lambda)$, can be transformed by means of elementary transformations of its columns to an equivalent matrix the elements of whose $\left(n_{1}+n_{2}+\cdots+n_{i-1}+1\right)$ st column, $i=1,2, \cdots, s$, have the common factor $\left(\lambda-x_{i}\right)^{n_{i}}$.

CoRollary 1. If $m=n,|A(\lambda)|$ is divisible by the characteristic determinant $\phi(\lambda)=|X-\lambda I|$, of a solution of (1), or may be identically zero.

* Böcher, op. cit., p. 262. 
CoROllary 2. If $m>n$ the determinant of an $n \times n$ matrix formed from $A(\lambda)$, by deleting any $m-n$ of its rows, is divisible by the characteristic determinant, $\phi(\lambda)=|X-\lambda I|$, of a solution of (1), or is identically zero.

If $m<n$ no such a simple corollary may be deduced from the theorem; this case will be taken up in Section III.

We shall now seek restrictions upon the degree and upon the number of elementary divisors of $|X-\lambda I|$ corresponding to the base $\left(x_{i}-\lambda\right)$.

Regard only the first $j-1, j \leqq n_{i}$, equations of (6). These may be looked upon as $m(j-1)$ linear and homogeneous equations in the $n(j-1)$ elements of $2_{{ }_{i}+k}, k=1,2, \cdots, j-1$, and may be written in the form

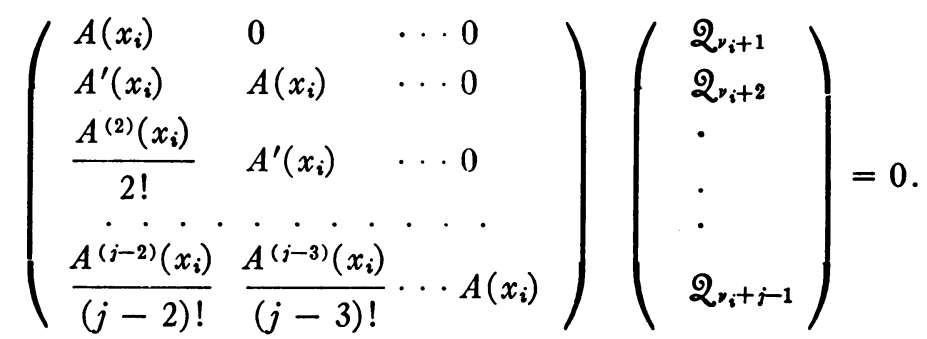

Let the rank of the matrix of the coefficients of this system be $r_{i, j-1}$. Since $Q$ is non-singular and $j \leqq n_{i}$, no $2_{v_{i}+k}, k=1,2, \cdots, j-1$, is zero, so that $r_{i, j-1}$ must be less than the number of elements in $2_{r_{i+k}}, k=1,2, \cdots, j-1$; that is,

$$
n(j-1)-r_{i, j-1}>0 .
$$

Suppose we have an entirely general set of matrices $2_{p_{i}+k}, k=1,2, \cdots$, $j-1$, satisfying (7), which we shall designate by

$$
2_{v_{i}+k}^{(j-1)} \quad(k=1,2, \cdots, j-1) .
$$

Let the number of arbitrary parameters among the elements of $2_{i_{i}+k}^{(j-1)}$ that are independent of those appearing in the elements of $2_{\nu_{i}+k}^{(j-1)}, 1 \leqq h<k$, be given by $e_{i k}^{(y-1)}$. Then the total number of independent parameters that may enter the matrices satisfying $(7)$ is

$$
\sum_{k=1}^{i-1} e_{i k}^{(j-1)}=n(j-1)-r_{i, j-1} .
$$

Now regard the system given by the first $j$ equations of (6) in the same way. The most general set of matrices satisfying this system is

$$
Q_{\nu,+k}^{(j)} \quad(k=1,2, \cdots, j) .
$$


Let $e_{i k}^{(f)}$ be the number of independent parameters that can occur in $2_{v_{i}+k}^{(f)}$ that are moreover independent of those in any matrix $2_{\nu_{i}+h}^{()}, 1 \leqq h<k$. Then, as above,

$$
\sum_{k=1}^{j} e_{i k}^{(j)}=n j-r_{i j}
$$

We shall show that

$$
r_{i j}-r_{i, j-1}<n, j \leqq n_{i},
$$

where we let $r_{i 0}=0$.

Evidently if $2_{\nu_{i}+k}^{(j-1)}, k=1,2, \cdots, j-1$, satisfy the first $j-1$ equations of (6) and if $2_{r_{i}+k}^{(j)}, k=1,2, \cdots, j$, satisfy the corresponding system of $j$ equations, then

$$
2_{v_{i+1}}^{(j)}, 2_{v_{i+2}}^{(j)}+2_{v_{i+1}}^{(j-1)}, \quad 2_{v_{i}+3}^{(j)}+2_{v_{i+2}}^{(j-1)}, \cdots, 2_{v_{i+j}}^{(j)}+2_{v_{i+j-1}}^{(j-1)}
$$

is again a set satisfying the first $j$ equations of (6) with absolutely no restrictions upon the arbitrary parameters that are in either set; hence we may conclude that

$$
e_{i k}^{(j)} \geqq e_{i, k-1}^{(j-1)} \quad(k=1,2, \cdots, j),
$$

where we let $e_{10}^{(j-1)}=0$. From this fact and from (8) and (9) we can conclude that

$$
n j-r_{i j} \geqq n(j-1)-r_{i, j-1} .
$$

The equality sign here cannot hold, for if it did then

$$
e_{i k}^{(j)}=e_{i, k-1}^{(j-1)} \quad(k=1,2, \cdots, j),
$$

and $e_{i 1}^{(j)}$ would be zero. That is, $\mathcal{Q}_{v_{i}+1}^{(j)}$ has no arbitrary parameters, and since $A\left(x_{i}\right) 2_{v_{i}+1}^{(1)}=0$ is a system of linear homogeneous equations in the elements of $2_{p_{i}+1}^{(j)}$ and if no arbitrary parameter occur among them, all must be zero. This is not possible for $j \leqq n_{i}$, since $Q$ is a non-singular matrix. Hence

$$
n j-r_{i j}>n(j-1)-r_{i, j-1},
$$

or

$$
r_{i j}-r_{i, j-1}<n, j \leqq n_{i} .
$$

THEOREM IV. The degree $n_{i}$ of the elementary divisor $\left(x_{i}-\lambda\right)^{n_{i}}$ of the characteristic determinant, $|X-\lambda I|$, of a solution of (1) cannot equal nor exceed the least positive integer, $j$, for which

$$
r_{i j}-r_{i, j-1} \geqq n .
$$


We can get still more general results. From (8), (9), and (10) we see that

$$
e_{i 1}^{(j)} \leqq n j-r_{i j}-\left(n(j-1)-r_{i, j-1}\right) .
$$

Now the number of matrices, $\mathscr{Q}_{v_{i}+1}^{\left(k_{i}\right)}, k_{i} \geqq j$, that are linearly independent cannot exceed $e_{i 1}^{(j)}$, where this is the number of arbitrary parameters in which the elements of $2_{i+1}^{(j)}$ are expressed. Corresponding to each elementary divisor $\left(x_{i}-\lambda\right)^{k_{i}}$, having a given base $\left(x_{i}-\lambda\right)$, we have a matrix $2_{v_{i}+1}^{\left(k_{i}\right)}$, and since $Q$ is non-singular the number of exponents $k_{i}$ that exceed $j$ cannot exceed

$$
e_{i 1}^{(j)} \leqq n-\left(r_{i j}-r_{i, j-1}\right) .
$$

This fact gives us the following important theorem:

TheOREM V. The number of elementary divisors $\left(x_{i}-\lambda\right)^{n_{i}}$ of the characteristic determinant of $X, a$ solution of (1), that are powers of the same linear factor, $x_{i}-\lambda$, and whose degree equals or exceeds $j$ cannot exceed

where we let $r_{i 0}=0$.

$$
n-\left(r_{i j}-r_{i, j-1}\right),
$$

In case $j=1$, we get Theorem II, likewise the preceding theorem may be derived as a special case of the above.

\section{Section III}

We shall now take up the study of solutions of (1) when $m<n$ or when the determinants of the $n \times n$ matrices formed from $A(\lambda)$ are all identically zero. The rank, $r$, of $A(\lambda)$, regarded as a $\lambda$-matrix, is then less than $n$. That is, the determinants of all matrices formed by the common elements of more than $r$ rows and columns of $A(\lambda)$ are identically zero, regarding $\lambda$ as a variable, whereas at least one $r \times r$ matrix so formed has as its determinant a polynomial in $\lambda$ not identically zero.* In the present section, we consequently consider the case where $r<n$; hence a solution of (1) may have arbitrary characteristic values, as is evident from considerations leading to Theorem 1.

Suppose that the characteristic determinant of a solution of (1) has the elementary divisors

$$
\left(\rho_{i}-\lambda\right)^{m_{i}} \quad(i=1,2, \cdots, \sigma),
$$

where $\rho_{i}, i=1,2, \cdots, \sigma$, are arbitrary save for certain values that will appear later, and the elementary divisors

$$
\left(x_{j}-\lambda\right)^{n_{j}} \quad(j=1,2, \cdots, s),
$$

* Bôcher, op. cit., p. 262. 
where $x_{i}, j=1,2, \cdots, s$, are constants. In particular, the latter may be certain ones of the exceptional values mentioned above, or those values, if any, of $\lambda$ for which the rank of $A(\lambda)$ is less than $r$. Hence the normal form of this solution is given by

where

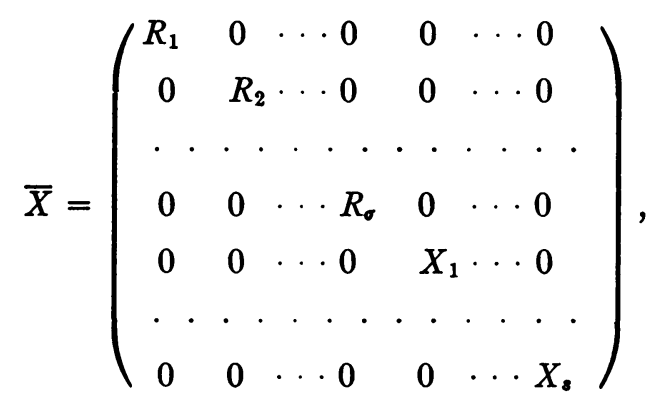

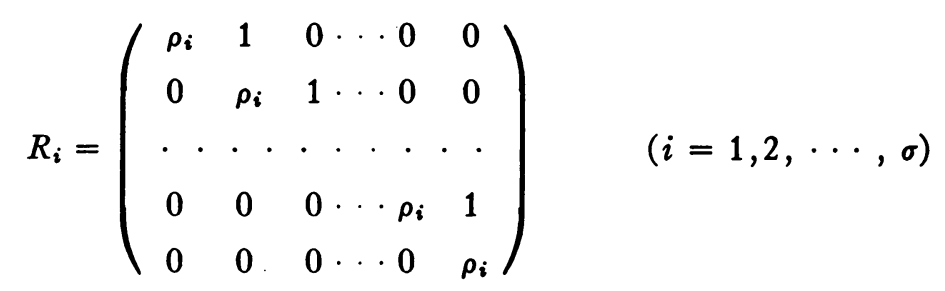

is of order $m_{i}$, and where

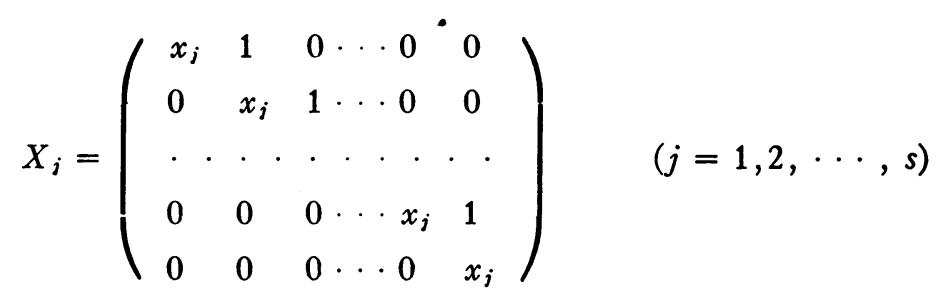

is of order $n_{j}$ and $\sum_{i=1}^{\sigma} m_{i}+\sum_{j=1}^{s} n_{j}=n$.

In this case again a non-singular $n \times n$ matrix, $Q$, must exist such that

$$
X=Q \bar{X} Q^{-!},
$$

but the elements $q_{h k}, h, k=1,2, \cdots, n$, of $Q$ are now rational functions of the parameters $\rho_{i}, i=1,2, \cdots, \sigma$; we shall assume, without restricting our problem in any way, that these elements are polynomials in the parameters, $\rho_{i}$, and that $Q^{-1}$ alone has rational functions as elements not all of which may be polynomials. Let

and

$$
\mu_{i}=m_{1}+m_{2}+\cdots+m_{i-1} \quad(i=1,2, \cdots, \sigma),
$$

$$
\nu_{j}=\mu_{\sigma}+m_{\sigma}+n_{1}+n_{2}+\cdots+n_{j-1} \quad(j=1,2, \cdots, s),
$$


where $\mu_{1}=0, \nu_{1}=m_{\sigma}+\mu_{\sigma}$; then by a procedure entirely parallel to that in Section I, we obtain the equations corresponding to (6) for the $m_{i}$ columns following the first $\mu_{i}$ or for the $n_{j}$ following the first $\nu_{j}$ of an equation corresponding to (3). Since the sequence in which elements $R_{i}$ and $X_{j}$ are written in $\bar{X}$ is immaterial, we shall write the system corresponding to (6) for $i=1$ with the subscript of $\rho$ suppressed. We have then

$$
\begin{aligned}
& A(\rho) \mathscr{Q}_{1}(\rho) \equiv 0 \\
& A^{\prime}(\rho) \mathscr{Q}_{1}(\rho)+A(\rho) \mathscr{Q}_{2}(\rho) \equiv 0, \\
& \ldots \ldots \ldots \ldots \\
& \frac{A^{\left(m_{1}-1\right)}(\rho)}{\left(m_{1}-1\right) !} \mathscr{Q}_{1}(\rho)+\frac{A^{\left(m_{1}-2\right)}(\rho)}{\left(m_{1}-2\right) !} 2_{2}(\rho)+\cdots+A(\rho) \mathscr{Q}_{m_{1}}(\rho) \equiv 0,
\end{aligned}
$$

where we are justified in writing $2_{k}(\rho), k=1,2, \cdots, m_{1}$, as matrices of a single column having elements that are polynomials in $\rho$ alone. The other parameters $\rho_{2}, \rho_{3}, \cdots, \rho_{\sigma}$ must drop out identically from these equations because the coefficients, $A^{(k)}(\rho), k=0,1, \cdots, m_{i}-1$, are independent of them. Evidently for the constant characteristic values the study reduces to that taken up in the preceding sections.

We can readily show that the first identity of (11),

$$
A(\rho) \mathscr{Q}_{1}(\rho) \equiv 0,
$$

is satisfied by only $n-r$ matrices:

$$
2_{1 j}(\rho) \quad(j=1,2, \cdots, n-r),
$$

where $r$ is the rank of $A(\lambda)$, and where $2_{1 j}(\rho)$ are such that the rank of the matrix

$$
\left(2_{11}(\rho), \mathscr{Q}_{12}(\rho), \cdots, \mathscr{Q}_{1, n-r}(\rho)\right)
$$

is $n-r$. Every other matrix $2_{1}(\rho)$ satisfying (12) is expressible linearly in terms of these $n-r$ in the following form:

$$
d(\rho) \mathscr{Q}_{1}(\rho) \equiv \sum_{j=1}^{n-r} d_{j}(\rho) \mathscr{Q}_{1 j}(\rho),
$$

where $d(\rho)$ and $d_{i}(\rho), i=1,2, \cdots, n-r$, are polynomials in $\rho$.

If $j$ of the parameters $\rho_{1}, \rho_{2}, \cdots, \rho_{\sigma}$ are identical, then $j$ columns of $Q$ must satisfy (12), hence the matrix formed by those $j$ columns will be of rank $j$ only if $j \leqq n-r$. 
THEOREM VI. The number of elementary divisors $\left(\rho_{i}-\lambda\right)^{m_{i}}, i=1,2, \cdots$, $\sigma$, of the characteristic determinant of a solution of (1) having the same base $\left(\rho_{i}-\lambda\right)$ or in which the bases $\left(\rho_{i}-\lambda\right), i=1,2, \cdots, \sigma$, may simultaneously be equals, cannot exceed $n-r$.

Suppose that $\mathscr{Q}_{1}(\lambda)=\left(q_{i 1}(\lambda)\right)$ satisfies the identity $A(\lambda) \mathscr{Q}_{1}(\lambda) \equiv 0$; then

$$
\sum_{j=1}^{n} a_{i j}(\lambda) q_{i 1}(\lambda) \equiv 0 \quad(i=1,2, \cdots, m),
$$

and differentiating with respect to $\lambda$, we have

or in matrices*

$$
\sum_{j=1}^{n} a_{i j}^{\prime}(\lambda) q_{i 1}(\lambda)+\sum_{j=1}^{n} a_{i j}(\lambda) q_{i 1}^{\prime}(\lambda) \equiv 0 \quad(i=1,2, \cdots, m),
$$

$$
A^{\prime}(\lambda) 2_{1}(\lambda)+A(\lambda) 2_{1}^{\prime}(\lambda) \equiv 0,
$$

where $A^{\prime}(\lambda)$ and $2_{1}^{\prime}(\lambda)$ are the matrices whose elements are the first derivatives with respect to $\lambda$ of the corresponding elements of $A(\lambda)$ and $2_{1}(\lambda)$ respectively. Continuing in this way to higher derivatives, and replacing $\lambda$ by $\rho$ in the first $m_{1}$ equations, including (12), so obtained, we see that

$$
\mathscr{Q}_{1}(\rho), \mathscr{Q}_{1}^{\prime}(\rho), \frac{\mathscr{Q}_{1}^{(2)}(\rho)}{2 !}, \ldots, \frac{\mathscr{Q}_{1}^{\left(m_{1}-1\right)}(\rho)}{\left(m_{1}-1\right) !}
$$

is a set of matrices satisfying (11), where $2_{1}(\rho)$ is a matrix satisfying the first equation of the system.

Let

$$
\mathscr{Q}_{11}(\rho), \mathscr{Q}_{12}(\rho), \cdots, \mathscr{Q}_{1 m_{1}}(\rho)
$$

be any $m_{1}$ matrices satisfying (12), and let

$$
2_{1}(\rho), \mathscr{Q}_{2}(\rho), \cdots, \mathscr{2}_{m_{1}}(\rho)
$$

be matrices satisfying (11), and suppose

$$
\begin{aligned}
& \mathscr{Q}_{1}(\rho) \equiv \mathscr{Q}_{11}(\rho), \\
& \mathscr{Q}_{i}(\rho) \equiv \delta_{j}(\rho)+\frac{2_{11}^{(j-1)}(\rho)}{(j-1) !} \quad\left(j=2,3, \cdots, m_{1}\right),
\end{aligned}
$$

where $S_{j}(\rho)$ will have a column of $n$ polynomial elements. By substitution in (11) we see that

$$
\delta_{j}(\rho) \quad\left(j=2,3, \cdots, m_{1}\right)
$$

* Frobenius, L.S., p. 16. Schlesinger, Vorlesungen über lineare Differentialgleichungen, 1908, p. 22. 
must satisfy a system like (11) with the last equation omitted, and that $S_{2}(\rho)$ must satisfy the first equation. Hence we may let

and suppose

$$
S_{2}(\rho)=2_{12}(\rho),
$$

$$
\mathcal{S}_{j}(\rho) \equiv \mathcal{V}_{j}(\rho)+\frac{\mathscr{Q}_{12}^{(j-2)}(\rho)}{(j-2) !} \quad\left(j=3,4, \cdots, m_{1}\right) ;
$$

then $\mathcal{T}_{3}(\rho)$ must again satisfy (12). Continuing in this way we find that

$$
\begin{array}{r}
\mathscr{Q}_{11}(\rho), \mathscr{2}_{11}^{\prime}(\rho)+\mathscr{Q}_{12}(\rho), \frac{2_{11}^{(2)}(\rho)}{2 !}+\mathscr{2}_{12}^{\prime}(\rho)+\mathscr{Q}_{13}(\rho), \\
\ldots, \frac{\mathscr{Q}_{11}^{\left(m_{1}-1\right)}(\rho)}{\left(m_{1}-1\right) !}+\frac{2_{12}^{\left(m_{1}-2\right)}(\rho)}{\left(m_{1}-2\right) !}+\cdots+\mathscr{Q}_{1 m_{1}}(\rho)
\end{array}
$$

is the general set of matrices satisfying (11), where $\mathscr{Q}_{1 j}(\rho), j=1,2, \cdots, m_{1}$, are general matrices satisfying only the first. The matrix of which these are the first, second, third, $\cdots$ and $m_{1}$ st column we shall designate by $Q_{m_{1}}\left(\rho_{1}\right)$; similarly for the remaining elementary divisors of $|X-\lambda I|$ we have

$$
Q_{m_{i}}\left(\rho_{i}\right) \quad(i=2,3, \cdots, \sigma),
$$

where $Q_{m_{i}}\left(\rho_{i}\right)$ is a matrix of $m$ rows and $m_{i}$ columns.

THEOREM VII. If

$$
X=Q \bar{X} Q^{-1}
$$

is a solution of (1) and $\bar{X}$ is its normal form, if $\left(\rho_{i}-\lambda\right)^{m_{i}}, i=1,2, \cdots, \sigma$, are the elementary divisors of the characteristic determinant of $X$, and if $2_{1 j}(\lambda)$, $j=1,2, \cdots, m_{1}$, are matrices satisfying the identity

$$
A(\lambda) \mathcal{Q}(\lambda) \equiv 0
$$

then the $m_{i}$ columns following the first $m_{1}+m_{2}+\cdots+m_{i-1}$ of $Q$ are given by

$$
\begin{aligned}
Q_{m_{i}}\left(\rho_{i}\right) \equiv\left(Q_{11}\left(\rho_{i}\right), \mathscr{Q}_{11}^{\prime}\left(\rho_{i}\right)+2_{12}\left(\rho_{i}\right), \cdots,\right. \\
\\
\left.\qquad \frac{\mathscr{2}_{11}^{\left(m_{i}-1\right)}(\rho)}{\left(m_{i}-1\right) !}+\frac{\mathscr{Q}_{12}^{\left(m_{i}-2\right)}\left(\rho_{i}\right)}{\left(m_{i}-2\right) !}+\cdots+2_{1 m_{i}}\left(\rho_{i}\right)\right)
\end{aligned}
$$

in which the matrices $\mathscr{Q}_{1 j}, j=1,2, \cdots, m_{i}$, are taken arbitrarily from those satisfying (12), and the values of $\rho_{i}, i=1,2, \cdots, \sigma$, that must be excepted are the values of these parameters for which the rank of

is less than $n$.

$$
Q=\left(Q_{m_{1}}\left(\rho_{1}\right), Q_{m_{2}}\left(\rho_{2}\right), \cdots, Q_{m_{\sigma}}\left(\rho_{\sigma}\right)\right)
$$


A sufficient condition that (1) have a solution is that $Q,|Q|$ not identically zero, may be constructed from the solutions of (12) in the manner given above. To do this may be impossible for solutions whose characteristic determinants have all elementary divisors of the kind $\left(\rho_{i}-\lambda\right)^{m_{i}}, i=1,2, \cdots$, $\sigma$, but (1) may have a solution in whose characteristic determinant the elementary divisors $\left(x_{j}-\lambda\right)^{n}, j=1,2, \cdots, s$, where $x_{j}$ are constants, are permitted. This possibly arises when the determinants of all $r \times r$ matrices formed from $A(\lambda)$ have the greatest common factor $p(\lambda)$, not a constant. Let the zeros of $p(\lambda)$ be $x_{j}, j=1,2, \cdots, s$; for these values the rank, $r_{j 1}$, of $A\left(x_{j}\right)$ is less than $r$, the rank of $A(\lambda)$. The columns that $Q$ must have permitting the characteristic values $x_{j}, j=1,2, \cdots, s$, must satisfy the equations (6) of the preceding sections. If the columns so determined for $x_{j}, j=1,2, \cdots, s$, and the columns satisfying (11) permit us to build up a non-singular matrix, $Q$, then a solution of (1) whose characteristic determinant has the corresponding elementary divisors

$$
\left(\rho_{i}-\lambda\right)^{m_{i}} \quad(i=1,2, \cdots, \sigma), \quad\left(x_{j}-\lambda\right)^{n_{j}} \quad(j=1,2, \cdots, s),
$$

exists. This is clarified by an example that will be given later. We can get a rather simple sufficient condition for the existence of solutions of (1) all of whose characteristic values are variables.

Suppose that $2(\lambda)$ such that

$$
A(\lambda) \mathcal{Q}(\lambda) \equiv 0
$$

and whose elements $q_{i}(\lambda), i=1,2, \cdots, n$, are polynomials of degree $q$ in $\lambda$, exists. Then

$$
2(\lambda) \equiv 2(\rho)+2^{\prime}(\rho)(\lambda-\rho)+\frac{2^{(2)}(\rho)}{2 !}(\lambda-\rho)^{2}+\cdots+\frac{2^{(q)}(\rho)}{q !}(\lambda-\rho)^{q},
$$

where $\mathscr{Q}^{(k)}(\lambda), k=0,1, \cdots, q$, is the matrix whose elements are the $k$ th derivatives of corresponding elements of $\mathscr{Q}(\lambda)$, is an identity in $\lambda$ and $\rho$. This identity may be written matricly as

$$
\mathscr{Q}(\lambda) \equiv\left(\mathcal{Q}(\rho), \mathscr{Q}^{\prime}(\rho), \cdots, \frac{\mathscr{Q}^{(q)}(\rho)}{q !}\right)\left(\begin{array}{c}
1 \\
\lambda-\rho \\
(\lambda-\rho)^{2} \\
\vdots \\
(\lambda-\rho)^{q}
\end{array}\right) .
$$


If here we replace $\lambda$ by $\rho_{i}, i=1,2, \cdots, n$, respectively, and regard the matrix, $Q$, having $2\left(\rho_{i}\right), i=1,2, \cdots, n$, as its columns, we have

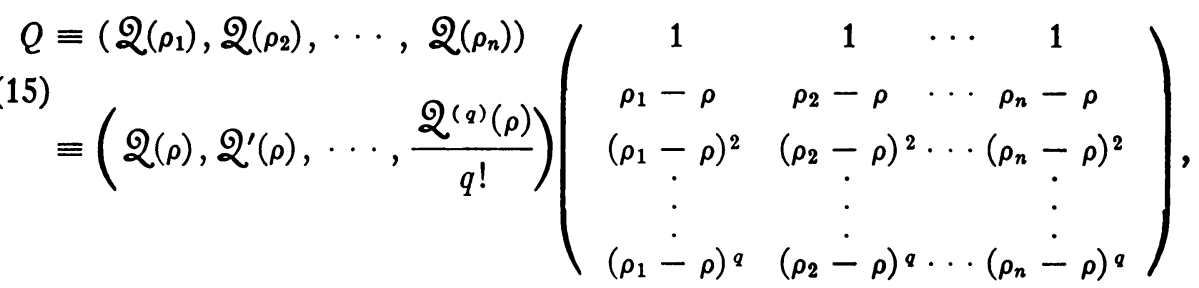

in which the first factor of the right member is an $n \times(q+1)$ matrix and the second is a $(q+1) \times n$ matrix. The rank of $Q$ cannot exceed the rank of either. factor; and if $q<n-1$, the rank of $Q$, whatever the parameters $\rho_{i}, i=1,2, \ldots$, $n$, may be is less than $n$. On the other hand, if $q$ equals or exceeds $n-1$, the first factor will certainly be of rank $n$ if the elements of Q $^{\prime}(\rho)$ are linearly independent, because the determinant of the matrix formed by its first $n$ columns is the Wronskian of those elements multiplied by a constant not zero. The second factor will be of rank $n$ if and only if $\rho_{i} \neq \rho_{i}, i \neq j$, and $q \geqq n-1$, for the determinant of the matrix formed by its first $n$ rows is the determinant of Vandermonde.

Now if the elements of $2(\lambda)$ are linearly independent and if $\rho_{i} \neq \rho_{j}$, $i \neq j$, then $Q$ is of rank $n$, for regarding the first factor of the right member of (15) as a $\lambda$-matrix with $\rho$ as the variable parameter and the second factor as elementary transformations, as defined earlier in the present section, upon the columns of the first, we see that this must be the case.*

With an appropriate change in the argument above, we can show that the matrix

$$
Q=\left(Q_{1}, Q_{2}, \cdots, Q_{\sigma}\right)
$$

where

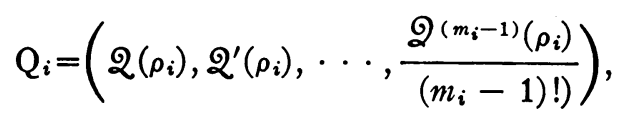

and $\rho_{i} \neq \rho_{j}$ for $i \neq j$, is of rank $n$ if the elements of $\mathscr{Q}(\lambda)$ are linearly independent. $\dagger$

\footnotetext{
* The degree, $q$, of the elements of $2(\lambda)$ must equal or exceed $n-1$, if they are linearly independent.

† See Eriksson, Sur une extension du déterminant de Vandermonde, Arkiv för Matematik, Astronomi, och Fysik, vol. 16 (1922), No. 13.
} 
ThEOREM VIII. A sufficient condition that equation (1) have a solution $X$ whose characteristic determinant has the elementary divisors

$$
\left(\rho_{i}-\lambda\right)^{m_{i}} \quad(i=1,2, \cdots, \sigma),
$$

where $\rho_{i}, i=1,2, \cdots, \sigma$, are all distinct but otherwise arbitrary parameters and where $\sum_{i=1}^{\sigma} m_{i}=n$, is that the equation

$$
A(\lambda) \mathcal{Q}(\lambda) \equiv 0
$$

be satisfied by a matrix $2(\lambda)$, whose elements $q_{j}(\lambda), j=1,2, \cdots, n$, are linearly independent. This condition is both necessary and sufficient for the existence of a solution of (1) whose characteristic determinant has the single elementary divisor $(\rho-\lambda)^{n}$, where $\rho$ is an arbitrary parameter.

This theorem may be extended in case the identity $A(\lambda) \mathcal{Q}(\lambda) \equiv 0$ is satisfied by $k$ matrices, $\mathscr{Q}_{k}(\lambda), k \leqq n-r$, such that

$$
\sum_{j=1}^{k} b_{j}(\lambda) \mathcal{Q}_{j}(\lambda) \equiv 0
$$

cannot be satisfied by polynomials $b_{i}(\lambda)$, not all identically zero, and such that the elements $q_{i j}(\lambda), i=1,2, \cdots, n$, of $\mathscr{Q}_{j}(\lambda)$ are linearly independent, to permit $k$ of the elementary divisors $\left(\rho_{i}-\lambda\right)^{m_{i}}$ to have the same base $\left(\rho_{i}-\lambda\right)$ or to take equal values.

Since the equation

$$
A(\lambda) \mathcal{Q}(\lambda) \equiv 0
$$

plays such an important part in case the rank of $A(\lambda)$ is less than $n$, we shall take up briefly a method of determining $2(\lambda)$ whose elements $q_{i}(\lambda), i=$ $1,2, \cdots, n$, are polynomials in $\lambda$ of degree not exceeding $j$. Such a matrix will always exist if $\jmath$ is taken sufficiently large, and is given by

$$
2(\lambda) \equiv 2_{0}+2_{1} \lambda+2_{2} \lambda^{2}+\cdots+2_{i} \lambda^{j},
$$

where $\mathscr{Q}_{k}, k=0,1, \cdots, j$, are matrices having a single column of $n$ constants, $q_{i k}, i=1,2, \cdots, n, k=0,1,2, \cdots, j$, and where

$$
q_{i}(\lambda)=q_{i 0}+q_{i 1} \lambda+\cdots+q_{i j} \lambda^{j} \quad(i=1,2, \cdots, m) ;
$$

not all $q_{i 0}, i=1,2, \cdots, n$, are zeros. Now

$$
A(\lambda)=A_{0} \lambda^{p}+A_{1} \lambda^{p-1}+\cdots+A_{p},
$$

and because (16) must be satisfied for all values of $\lambda$, we have at once the equations*

* Compare Kronecker, Vorlesungen über die Theorie der Determinanten, vol. 1, 1903, p. 363. 


$$
\begin{aligned}
& A_{p} 2_{0}=0 \text {, } \\
& A_{p-1} 2_{0}+A_{p} 2_{1}=0 \text {, } \\
& A_{0} 2_{0}+A_{1} 2_{1}+\cdots+A_{j} 2_{j}=0 \text {, } \\
& A_{0} \mathscr{Q}_{1}+\cdots+A_{j-1} \mathscr{2}_{j}=0 \text {, } \\
& A_{0} 2_{j}=0 .
\end{aligned}
$$

This is a system of $(p+j+1) m$ linear and homogeneous equations in the $(j+1) n$ coefficients of the elements of $2(\lambda)$. The degree $j$ must be taken sufficiently large so that the rank of the matrix of the coefficients is less than $(j+1) n$. This is always possible since the rank, $r$, of $A(\lambda)$ is less than $n$. In fact, $j$ can be so chosen that the rank of the system does not exceed $n j+r$, then the general solution of (17) gives us a fundamental set of polynomials $2_{j}(\lambda), j=1,2, \cdots, n-r$, in terms of which all others satisfying (12) may be expressed linearly with polynomial coefficients. Moreover, from (17) it is evident that if either $A_{0}$ or $A_{p}$ is of rank $n$ then $2(\lambda)$ satisfying (16) must be identically zero, hence equation (1) may have solutions having variable characteristic values only when the rank of $A_{0}$ and of $A_{p}$ is less than $n$.

We shall illustrate the above theory with two examples:

EXAMple 1.

Here

$$
(1,0,1) X^{2}+(-3,2,-2) X+(0,2,-3)=0 .
$$

$$
A(\lambda)=\left(\lambda^{2}-3 \lambda, 2 \lambda+2, \lambda^{2}-2 \lambda-3\right)
$$

and according to equations (17) a solution of the first degree in $\lambda$ of (12) is

$$
2(\lambda)=q_{0}\left(\begin{array}{c}
\lambda+1 \\
0 \\
-\lambda
\end{array}\right)+q_{1}\left(\begin{array}{c}
0 \\
\lambda-3 \\
-2
\end{array}\right),
$$

where $q_{0}$ and $q_{1}$ are arbitrary constants. The general solution of (12) is then

$$
b_{0}(\lambda)\left(\begin{array}{c}
\lambda+1 \\
0 \\
-\lambda
\end{array}\right)+b_{1}(\lambda)\left(\begin{array}{c}
0 \\
\lambda-3 \\
-2
\end{array}\right),
$$

where $b_{0}(\lambda)$ and $b_{1}(\lambda)$ are polynomials in $\lambda$. If we let $b_{0}(\lambda) \equiv \lambda$ and $b_{1}(\lambda) \equiv 1$ we have

$$
2(\lambda)=\left(\begin{array}{c}
\lambda^{2}+\lambda \\
\lambda-3 \\
-\lambda^{2}-2
\end{array}\right),
$$


a matrix satisfying (12) whose elements are linearly independent. Hence by Theorem VIII the given equation has the following solutions:

$$
\begin{aligned}
& X_{1}=Q_{1}\left(\begin{array}{lll}
\rho & 1 & 0 \\
0 & \rho & 1 \\
0 & 0 & \rho
\end{array}\right) Q_{1}^{-1}, \quad Q_{1}=\left(\begin{array}{ccc}
\rho^{2}+\rho, & 2 \rho+1, & 1 \\
\rho-3, & 1, & 0 \\
-\rho^{2}-2, & -2 \rho, & -1
\end{array}\right) ; \\
& X_{2}=Q_{2}\left(\begin{array}{lll}
\rho_{1} & 1 & 0 \\
0 & \rho_{1} & 0 \\
0 & 0 & \rho_{2}
\end{array}\right) Q_{2}^{-1}, \quad Q_{2}=\left(\begin{array}{rrr}
\rho_{1}^{2}+\rho_{1}, & 2 \rho_{1}+1, & \rho_{2}{ }^{2}+\rho_{2} \\
\rho_{1}-3, & 1, & \rho_{2}-3 \\
-\rho_{1}{ }^{2}-2, & -2 \rho_{1}, & -\rho_{2}{ }^{2}-2
\end{array}\right), \\
& \rho_{1} \neq \rho_{2} ; \\
& X_{3}=Q_{3}\left(\begin{array}{lll}
\rho_{1} & 0 & 0 \\
0 & \rho_{2} & 0 \\
0 & 0 & \rho_{3}
\end{array}\right) Q_{3}^{-1}, Q_{3}=\left(\begin{array}{rrr}
\rho_{1}^{2}+\rho_{1}, & \rho_{2}{ }^{2}+\rho_{2}, & \rho_{3}^{2}+\rho_{3} \\
\rho_{1}-3, & \rho_{2}-3, & \rho_{3}-3 \\
-\rho_{1}^{2}-2, & -\rho_{2}{ }^{2}-2, & -\rho_{3}^{2}-2
\end{array}\right),
\end{aligned}
$$

however, according to Theorem VI elementary divisors having the same base may occur, at most, twice in the characteristic determinants of solutions, since the rank of $A(\lambda)$ is unity. Such solutions are

where

$$
X_{4}=Q_{4}\left(\begin{array}{ccc}
\rho_{1} & 1 & 0 \\
0 & \rho_{1} & 0 \\
0 & 0 & \rho_{2}
\end{array}\right) Q_{4}^{-1}
$$

$$
Q_{4}=\left(\begin{array}{ccc}
\rho_{1}+1 & 1 & 0 \\
0 & 0 & \rho_{2}-3 \\
-\rho_{1} & -1 & -2
\end{array}\right), \quad \text { or } \quad Q_{4}=\left(\begin{array}{ccc}
0 & 0 & \rho_{2}+1 \\
\rho_{1}-3 & 1 & 0 \\
-2 & 0 & -\rho_{2}
\end{array}\right) \text {; }
$$

$\rho_{2}$ in the first cannot equal 3 , in the second -1 ;

or

$$
\begin{aligned}
& X_{5}=Q_{5}\left(\begin{array}{ccc}
\rho_{1} & 0 & 0 \\
0 & \rho_{2} & 0 \\
0 & 0 & \rho_{3}
\end{array}\right) Q_{5}^{-1}, \\
& Q_{5}=\left(\begin{array}{ccc}
\rho_{1}+1 & 0 & \rho_{3}+1 \\
0 & \rho_{2}-3 & 0 \\
-\rho_{1} & -2 & -\rho_{3}
\end{array}\right), \begin{array}{l}
\rho_{1} \neq \rho_{3}, \\
\rho_{2} \neq 3,
\end{array}
\end{aligned}
$$

$$
Q_{5}=\left(\begin{array}{ccc}
0 & \rho_{2}+1 & 0 \\
\rho_{1}-3 & 0 & \rho_{3}-3 \\
-2 & -\rho_{2} & -2
\end{array}\right), \begin{aligned}
& \rho_{1} \neq \rho_{3} \\
& \rho_{2} \neq-1
\end{aligned}
$$


the last two solutions, $X_{4}$ and $X_{5}$, are less restrictive than are $X_{2}$ and $X_{3}$.

EXAMPLE 2.

$$
\left(\begin{array}{rrr}
1 & 0 & 1 \\
-1 & 0 & 0
\end{array}\right) X^{2}+\left(\begin{array}{rrr}
-3 & 1 & -3 \\
3 & -1 & -1
\end{array}\right) X+\left(\begin{array}{rrr}
2 & 2 & 2 \\
-2 & -2 & 1
\end{array}\right)=0
$$

This equation has no solutions all of whose characteristic values are variables, since the equation $A(\lambda) 2(\lambda) \equiv 0$ is satisfied only by

$$
2(\lambda)=b(\lambda)\left(\begin{array}{c}
\lambda+2 \\
-\lambda^{2}+3 \lambda-2 \\
0
\end{array}\right),
$$

whose elements are not linearly independent. Theorem VIII is not satisfied; however the rank of $A(\lambda)$ is unity when $\lambda=1$, and for this value of $\lambda$ we have the following equations corresponding to (5):

$$
\begin{aligned}
& \left(\begin{array}{rrr}
0 & 3 & 0 \\
0 & -3 & 0
\end{array}\right)\left(\begin{array}{l}
q_{1} \\
q_{2} \\
q_{3}
\end{array}\right)=0, \\
& \left(\begin{array}{rrr}
-1 & 1 & -1 \\
1 & -1 & -1
\end{array}\right)\left(\begin{array}{l}
q_{1} \\
q_{2} \\
q_{3}
\end{array}\right)+\left(\begin{array}{rrr}
0 & 3 & 0 \\
0 & -3 & 0
\end{array}\right)\left(\begin{array}{l}
r_{1} \\
r_{2} \\
r_{3}
\end{array}\right)=0, \\
& \left(\begin{array}{rrr}
1 & 0 & 1 \\
-1 & 0 & 0
\end{array}\right)\left(\begin{array}{l}
q_{1} \\
q_{2} \\
q_{3}
\end{array}\right)+\left(\begin{array}{rrr}
-1 & 1 & -1 \\
1 & -1 & -1
\end{array}\right)\left(\begin{array}{l}
r_{1} \\
r_{2} \\
r_{3}
\end{array}\right)+\left(\begin{array}{lll}
0 & 3 & 0 \\
0 & -3 & 0
\end{array}\right)\left(\begin{array}{l}
s_{1} \\
s_{2} \\
s_{3}
\end{array}\right)=0 .
\end{aligned}
$$

The first of these is satisfied by

the first two by

$$
2_{1}=\left(\begin{array}{c}
q_{1} \\
0 \\
q_{3}
\end{array}\right)
$$

all three by

$$
Q_{1}=\left(\begin{array}{c}
3 r_{2} \\
0 \\
0
\end{array}\right), \quad Q_{2}=\left(\begin{array}{c}
r_{1} \\
r_{2} \\
r_{3}
\end{array}\right)
$$

$$
\mathscr{Q}_{1}=\left(\begin{array}{c}
3 r_{2} \\
0 \\
0
\end{array}\right), \quad 2_{2}=\left(\begin{array}{c}
4 r_{2}+3 s_{2} \\
r_{2} \\
0
\end{array}\right), \quad 2_{3}=\left(\begin{array}{c}
s_{1} \\
s_{2} \\
s_{3}
\end{array}\right) \text {, }
$$


where the constants are arbitrary. The given equation then has solutions:

$$
\begin{aligned}
& X_{1}=Q_{1}\left(\begin{array}{ccc}
\rho & 1 & 0 \\
0 & \rho & 0 \\
0 & 0 & 1
\end{array}\right) Q_{1}^{-1}, Q_{1}=\left(\begin{array}{ccc}
\rho+2 & 1 & q_{1} \\
-\rho^{2}+3 \rho-2 & -2 \rho+3 & 0 \\
0 & 0 & q_{3}
\end{array}\right) \\
& q_{3}\left(\rho^{2}+4 \rho-8\right) \neq 0 \text {; } \\
& X_{2}=Q_{2}\left(\begin{array}{ccc}
\rho_{1} & 0 & 0 \\
0 & \rho_{2} & 0 \\
0 & 0 & 1
\end{array}\right) Q_{2}^{-1}, Q_{2}=\left(\begin{array}{ccc}
\rho_{1}+2 & \rho_{2}+2 & q_{1} \\
-\rho_{1}^{2}+3 \rho_{1}-2 & -\rho_{2}^{2}+3 \rho_{2}-2 & 0 \\
0 & 0 & q_{3}
\end{array}\right) \text {, } \\
& q_{2}\left(\rho_{1}-\rho_{2}\right)\left(\rho_{1} \rho_{2}+2 \rho_{1}+2 \rho_{2}-8\right) \neq 0 ; \\
& X_{3}=Q_{3}\left(\begin{array}{lll}
\rho & 0 & 0 \\
0 & 1 & 1 \\
0 & 0 & 1
\end{array}\right) Q_{3}^{-1}, \quad Q_{3}=\left(\begin{array}{ccc}
\rho+2 & 3 r_{2} & r_{1} \\
-\rho^{2}+3 \rho-2 & 0 & r_{2} \\
0 & 0 & r_{3}
\end{array}\right) \text {, } \\
& r_{2} r_{3}\left(\rho^{2}-3 \rho+2\right) \neq 0 \text {; } \\
& X_{4}=Q_{4}\left(\begin{array}{lll}
\rho & 0 & 0 \\
0 & 1 & 0 \\
0 & 0 & 1
\end{array}\right) Q_{4}^{-1}, \quad Q_{4}=\left(\begin{array}{ccc}
\rho+2 & q_{1} & r_{1} \\
-\rho^{2}+3 \rho-2 & 0 & 0 \\
0 & q_{3} & r_{3}
\end{array}\right) \\
& \left(q_{1} r_{3}-q_{3} r_{1}\right)\left(\rho^{2}-3 \rho+2\right) \neq 0 \text {; } \\
& X_{5}=Q_{5}\left(\begin{array}{ccc}
1 & 1 & 0 \\
0 & 1 & 1 \\
0 & 0 & 1
\end{array}\right) Q_{5}^{-1}, \quad Q_{5}=\left(\begin{array}{ccc}
3 r_{2} & 4 r_{2}+3 s_{2} & s_{1} \\
0 & r_{2} & s_{2} \\
0 & 0 & s_{3}
\end{array}\right) \\
& r_{2} s_{3} \neq 0 \text {; }
\end{aligned}
$$

where $\rho_{1}, \rho_{2}, q_{1}, q_{3}, r_{1}, r_{2}, r_{3}, s_{1}, s_{2}, s_{3}$ are entirely arbitrary save that the determinant of $Q$ in each case shall be different from zero. The given equation has no solutions whose characteristic determinants have as elementary divisors

$$
\begin{gathered}
\left(-2 \pm 2 \cdot 3^{1 / 2}-\lambda\right)^{2},(1-\lambda), \\
(2-\lambda),(1-\lambda)^{2}, \\
2-\lambda, 1-\lambda, 1-\lambda,
\end{gathered}
$$

because for each of these groups equations (6) lead to a singular $Q$, nor as ele:mentary divisors

because of Theorem II.

$$
1-\lambda, 1-\lambda, 1-\lambda
$$

University Extension Division, UNIVERSITY OF WISCONSIN, MilwaukeE, Wis. 